\title{
Erratum
}

\section{We apologize}

In issue No. 6 of the journal for 1999, the 3rd line from the top on p. 375 should read: "Concentration of primary products..." ("polymeric" was erroneously printed). 